\title{
Pathogenesis of invasive micropapillary carcinoma: role of MUC1 glycoprotein
}

\author{
Hind Nassar ${ }^{1,2}$, Vishali Pansare ${ }^{1,2}$, Hongquan Zhang ${ }^{1,2}$, Minxing Che $^{1,2}$, Wael Sakr ${ }^{1,2}$, \\ Rouba Ali-Fehmi ${ }^{1,2}$, David Grignon ${ }^{1,2}$, Fazlul Sarkar ${ }^{1,2}$, Jeanette Cheng ${ }^{3}$ and Volkan Adsay ${ }^{1,2}$ \\ ${ }^{1}$ Department of Pathology, Wayne State University, Harper University Hospital Detroit, MI, USA; ${ }^{2}$ Karmanos \\ Cancer Institute, Detroit, MI, USA and ${ }^{3}$ Department of Pathology, Providence Hospital, Detroit, MI, USA
}

\begin{abstract}
Invasive micropapillary carcinoma, a tumor with highly infiltrative characteristics is defined by a distinctive cleft formation around the neoplastic cell clusters which is presumably a result of the detachment of the cells from the stroma due to as yet undetermined factors. Ultrastructural examination performed on a handful of cases demonstrated an unexpected secretory activity in the stroma-facing surface of the cells. MUC1 is a glycoprotein typically expressed in the apical surface of normal epithelial cells, responsible for maintaining lumen formation. In conventional adenocarcinomas, MUC1 expression is largely intracytoplasmic, intercellular, or apical (in glandular areas). The MUC1 expression pattern was investigated by immunohistochemical staining in invasive micropapillary carcinoma of breast $(n=11)$, pancreas $(n=5)$, gynecologic tract $(n=11)$ and urinary bladder $(n=10)$. The results were contrasted with the staining pattern in conventional carcinomas of the same organs $(n=202)$. In all invasive micropapillary carcinoma, MUC1 expression was predominantly in the stroma-facing surface of the cell clusters (basal), accentuating the outlines of the micropapillary units by forming a distinct band on this surface. In conventional carcinoma the labeling was mostly apical in areas with lumen formation and intracytoplasmic and intercellular in the poorly differentiated areas. In conclusion, in the micropapillary pattern of invasive carcinoma, the expression of MUC1, is largely limited to the basal surface of the cells in contrast to conventional carcinomas in which MUC1 is largely apical, intracytoplasmic or intercellular. This provides support for the reversal of cell orientation as an important factor of the morphogenesis and possibly the pathogenesis of invasive micropapillary carcinoma. Since MUC1 is known to have a role in lumen formation, and has an inhibitory role in the cell to stroma interaction, it is conceivable that it is a key factor in the detachment of cells from stroma allowing for the dissection of the connective tissue and easing the spread of cells.
\end{abstract}

Modern Pathology (2004) 17, 1045-1050, advance online publication, 21 May 2004; doi:10.1038/modpathol.3800166

Keywords: MUC1; micropapillary; carcinoma; polarity

Invasive micropapillary carcinoma is the name given to a distinctive pattern of carcinoma characterized by cleft formation around neoplastic cell clusters (Figure 1). ${ }^{1,2}$ Tumors with this morphology, even when focal in otherwise conventional carcinoma, are characterized by a high degree of lymphotropism. ${ }^{2-7}$ Electron microscopy examination in a handful of invasive micropapillary carcinoma cases demonstrated an unexpected secretory activity in the stroma-facing surface of the tumor cells suggesting a reversal in cell polarity in this particular type of tumor. ${ }^{2}$

MUC1 is a type of mucin (a surface glycoprotein) encoded by the MUC1 gene, located on chromosome

Correspondence: Dr H Nassar, MD, Department of Pathology, Harper University Hospital, 3990 John R, Detroit, MI 48201, USA. E-mail: hnassar@dmc.org

Received 31 October 2003; revised 29 December 2003; accepted 5 January 2004; published online 21 May 2004
1 (1q21) ${ }^{8-10}$ MUC1 is expressed on the cell membranes in a variety of normal tissues where it is exclusively present on the apical surface of epithelial cells.

The purpose of our study is to test the hypothesis of reverse cellular polarization in invasive micropapillary carcinoma by investigating the cellular distribution of MUC1, the apical glycoprotein, in invasive micropapillary carcinoma from different organs, and contrast it with the expression patterns of MUC1 in the conventional carcinomas of the same sites.

\section{Material and methods}

Cases

In all, 37 cases of invasive micropapillary carcinoma and 202 cases of conventional carcinomas were 
1046

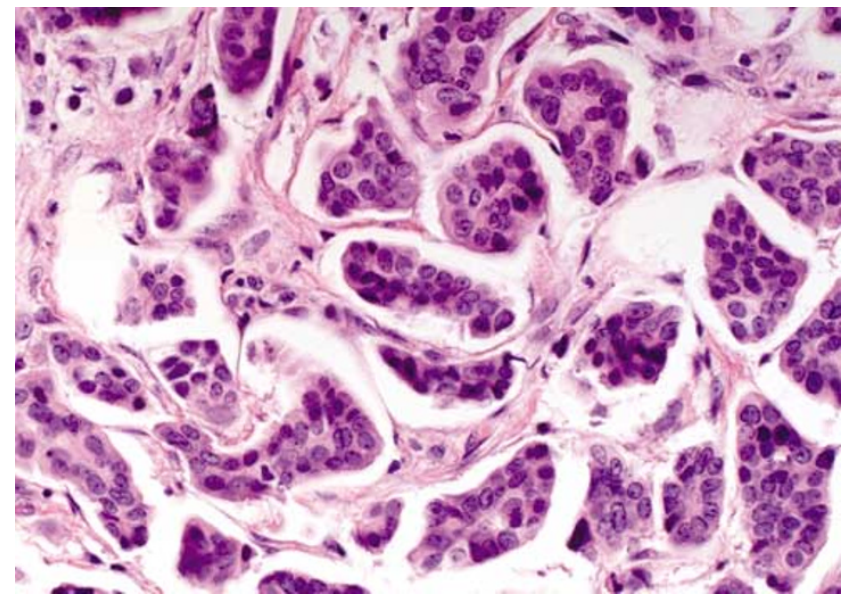

Figure 1 Invasive micropapillary carcinoma of the breast. The neoplastic cell clusters are surrounded by clear spaces.

gathered from the pathology files of the Karmanos Cancer Institute and Wayne State University. Of the invasive micropapillary carcinoma cases, 11 did arise in breast, five in pancreas, 11 in the ovary (invasive micropapillary/low-grade serous carcinomas), and 10 in the urinary bladder.

Of the conventional carcinomas, 45 were in breast (30 invasive ductal and 15 invasive lobular carcinomas), 136 were in pancreas (conventional pancreatobiliary adenocarcinomas), 11 were in the ovary (high-grade serous papillary carcinomas), and 10 were in the urinary bladder (nine invasive urothelial and one high-grade papillary urothelial carcinomas).

\section{Immunohistochemical Stains}

The immunohistochemical stains were performed by the avidin-biotin peroxidase method. The commercial antibody MUC1 (clone Ma695) was purchased from Vector laboratories (Burlingame, CA, USA). After deparaffinization and blocking of endogenous peroxidase, tissue sections were steamed in $10 \mathrm{M}, \mathrm{pH} 6.0$, citrate buffer for $20 \mathrm{~min}$ and allowed to stand in the lot buffer for an additional 20 min. MUC1 antibody diluted 1:1000, was incubated with the tissue sections for $60 \mathrm{~min}$. Biotinylated anti-mouse and avidin-biotin complex were applied for $10 \mathrm{~min}$ each (Vector, Burlingame, CA; dilution 1:200 for the biotinylated anti-mouse). After color development with 3-amino-9-ethylcarbazole, sections were counterstained with hematoxylin. Benign and neoplastic breast tissues were used as controls.

\section{Evaluation of the Labeling}

The distribution pattern of MUC1 was evaluated in each case as luminal (rimming the apical membrane of the cell in glandular or cystic areas), intercellular (highlighting the intercellular aspect of the cytoplasmic membrane), basal (labeling the surface of the cell facing the stroma), and intracytoplasmic. We also noted the percentage of positive cells and classified the tumor arbitrarily accordingly as focally $(<50 \%$ of the cells) or diffusely $(>50 \%$ of the cells) staining for MUC1. Fisher's exact test was used to compare the location of expression of MUC1 between the invasive micropapillary and conventional carcinomas.

\section{Results}

\section{Staining in the Normal Tissue}

In the normal breast tissue MUC1 labeling was limited to the apical surface of the ducts, forming a thin band (Figure 2). Similar pattern was seen in the intralobular small ductules in pancreas (those in the close proximity to the centroacinar area, lined by small cuboidal cells with minimal cytoplasm). The interlobular ducts, acini, and islets were negative for MUC1.

In normal urothelium MUC1 expression was consistently strong and diffuse in the apical membranes of the umbrella cell layer. Focal cytoplasmic staining was also seen in urothelial cells of deeper layers and Von Brunn's nests. The amount of staining was variable between different cases.

In ovary, MUC1 was expressed in the cell apex of the germinal coelomic epithelium when it had plump cells, as well as in the luminal surface of cells lining benign epithelial inclusion cysts and endometriosis glands and cysts, when present.

\section{Staining in the Invasive Micropapillary Carcinoma}

In all carcinomas with invasive micropapillary morphology, invariably and regardless of the site,

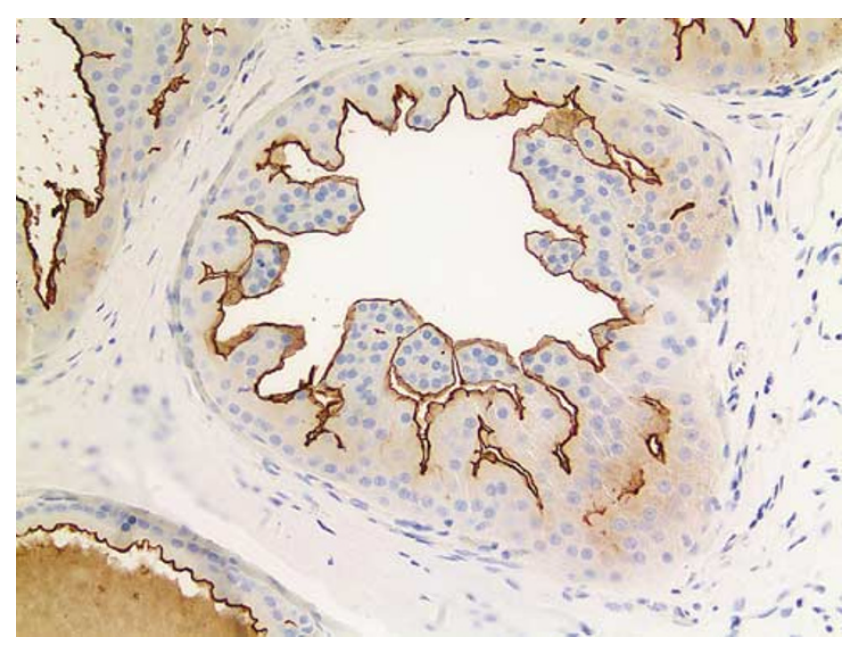

Figure 2 MUC1 in benign breast tissue with apocrine metaplasia. Band-like staining on the luminal (apical) surface of the ducts. 

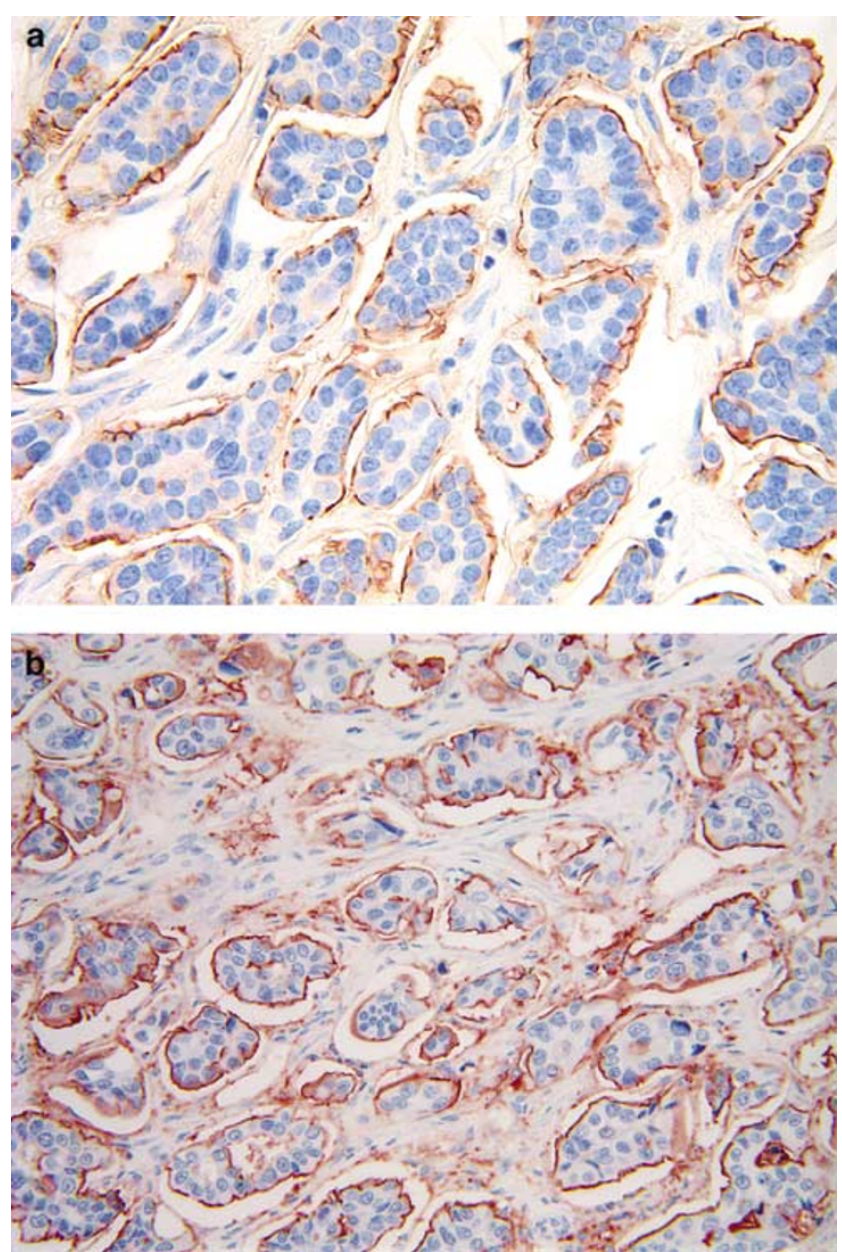

Figure 3 MUC1 in invasive micropapillary carcinoma. Band-like labeling present on the stroma-facing (basal) surface on the neoplastic cell clusters. (a) Invasive micropapillary carcinoma of the breast. (b) Invasive micropapillary carcinoma of the urinary bladder.

MUC1 expression was strongly and diffusely present in the stroma-facing (basal) surface of the neoplastic cell clusters, consisting of a thin band of staining (Figure 3). In few cases (three in breast, and six in urinary bladder) there was in addition a focal intracytoplasmic staining. In two invasive micropapillary carcinoma cases arising in breast, when lumens were present within the clusters, an apical rimming was also seen in addition to the basal staining.

\section{Staining in Conventional Adenocarcinoma}

Overall the patterns of staining were different between invasive micropapillary and conventional carcinoma $(P<0.001)$. None of the conventional carcinomas displayed a basal labeling for MUC1 glycoprotein $(P<001)$. In breast, the staining was cytoplasmic in 34 cases $(75 \%)$, luminal in eight $(18 \%)$, and intercellular in $28(62 \%)$. In pancreas, 88

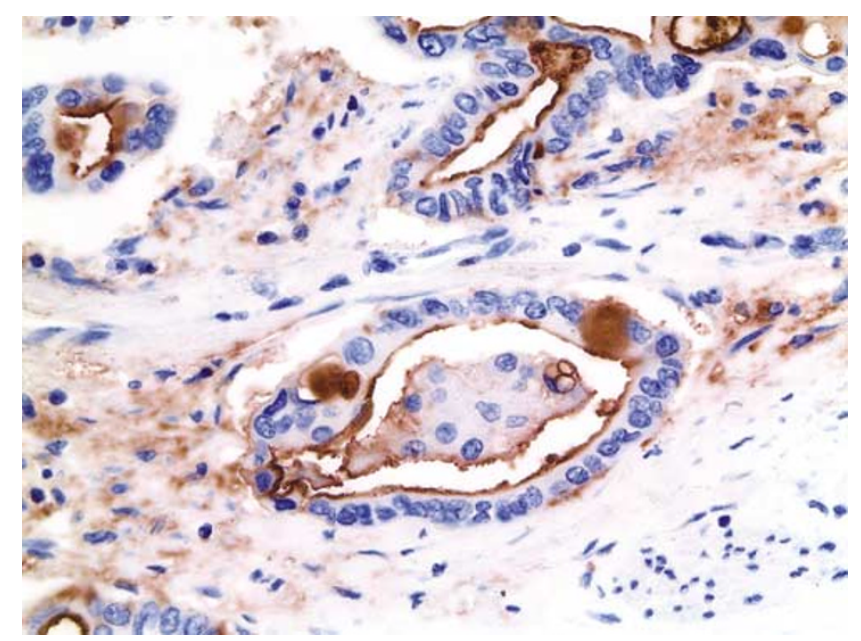

Figure 4 MUC1 in conventional ductal adenocarcinoma of the pancreas. Luminal (apical) and intracytoplasmic staining in glandular structures.

adenocarcinomas had intracytoplasmic labeling (65\%), 102 had luminal staining $(75 \%)$, and an intercellular staining was seen in 108 tumors $(80 \%)$ (Figure 4). In these organs (breast and pancreas) the staining was usually diffuse. In all urothelial carcinomas MUC1 expression was both intracytoplasmic and intercellular. This expression was usually focally present. In the high-grade serous ovarian carcinomas all tumors showed focal luminal, intracytoplasmic, and intercellular labeling.

\section{Discussion}

Invasive micropapillary morphology is being increasingly recognized as a prognostically important pattern of adenocarcinoma occurring in various organs including breast, urinary bladder, ovary, and lung. ${ }^{2-6,11-13}$ Except in the ovary, where it is considered a low-grade carcinoma and displays an indolent behavior-unlike the conventional/highgrade serous carcinoma, ${ }^{12}$ invasive micropapillary carcinoma has been associated with a high degree of aggressiveness in other organs, manifested by high stage at presentation, massive lymph node metastasis and extensive lymphovascular invasion. ${ }^{2,4,6,7,11,13}$ There is no clear explanation for the morphogenesis of this tumor or on how this particular morphology affects its behavior. LunaMore et $a{ }^{2}{ }^{2}$ in a study of a series of invasive micropapillary carcinoma of breast, demonstrated an unexpected secretory activity towards the surrounding stroma in two tumors, showing by electron microscopy, the presence of microvilli on the cell surface facing the stroma. Furthermore, they demonstrated by special stains, in some of their cases, the presence of a small amount of mucinous secretions in the clear spaces surrounding the epithelial clusters. In keeping with these findings, our study 
shows that MUC1, a glycoprotein normally located in the apical cell surface of normal glandular epithelium, has a highly aberrant expression in invasive micropapillary carcinoma where it is localized predominantly in the stroma-facing surface of the cells.

MUC1 is a high molecular weight, heavily glycosylated transmembrane glycoprotein, expressed in the apical surface of a wide variety of epithelial tissues. ${ }^{14}$ It is composed of a cytoplasmic tail of 69 aa and a large extracellular domain that consists of a variable number of tandem repeats rich in serine and threonine residues. ${ }^{9,15,16}$ This domain undergoes specific O-glycosylation to generate a broad range of glycosylated variants of the MUC1 molecule differing between various organ sites. ${ }^{9,16}$ The physical and chemical properties of this extracellular domain-large size, rigidity, and negative charge-are believed to be responsible for the ability of MUC1 to maintain lumen integrity in normal glandular tissues. This is probably due to the fact that this glycoprotein could counteract the interaction of much smaller membrane associated molecules, including adhesion molecules such as $\beta$ integrin and E-cadherin. ${ }^{17}$ Moreover, in vitro studies showed that increasing MUC1 expression results in decreased adhesion between adjacent cells and between cells and extracellular matrix (ECM). ${ }^{18-20}$ It also results in contraction of collagen type I matrix and altered expression of epithelial cytokeratins. ${ }^{21}$ Therefore, the consistent expression of MUC1 in invasive micropapillary carcinoma on the stromafacing rather than the apical surface of the cellsindependent of the location of the tumor-may be responsible at least in part, for the detachment of the cells from the stroma, one of the main entity-defining features of invasive micropapillary carcinoma.

Although, increased expression of MUC1 in conventional carcinoma correlated with low-grade cytology and good prognosis, ${ }^{22-26}$ aberrant expression such as intracytoplasmic localization was shown by multiple studies to be associated with poor outcome, independent of tumor differentiation. ${ }^{26-30}$ Intercellular/circumferential labeling was associated with a high incidence of lymph node metastases as well. ${ }^{28}$ Interestingly, none of these labeling patterns (intracytoplasmic or intercellular) is seen in invasive micropapillary carcinoma despite the high propensity of the tumor for lymph node metastases and its poor prognosis. Our finding in invasive micropapillary carcinoma, however, provides further evidence that any aberrant localization of MUC1 in the tumor cells (intracytoplasmic, intercellular, or basal) confers to the tumor a worse prognosis and possibly a high propensity to disseminate. Whether the basal expression of MUC1 acts as an independent prognostic factor in invasive micropapillary carcinoma is not clear.

Our findings also confirm the prior impression that invasive micropapillary carcinoma is character- ized by an abnormality in cell polarity that occurs in a fashion that is not seen in conventional carcinoma. In fact, in invasive micropapillary carcinoma the surface of the tumor cells that faces the stroma acquires apical secretory properties. This was evidenced earlier by electron microscopy ${ }^{2}$ and in the current study by immunohistochemical staining for MUC1. It is noteworthy that colloid (mucinous) carcinoma of the breast and pancreas are characterized by similar findings, that is, a band-like staining on the surface of the cell clusters for apical glycoproteins (MUC1 in breast and CEA in pancreas) and the lack of basement membrane and presence of microvilli at the basal surface of the cells at the ultrastructural level. ${ }^{31}$ A major molecular difference between invasive micropapillary carcinoma (a tumor with a high propensity for early dissemination) and colloid carcinoma (a tumor with a much better prognosis than conventional carcinoma), ${ }^{32-35}$ however, is the positivity of the latter for MUC2, the gelforming intestinal mucin, and the total lack of MUC2 expression in invasive micropapillary carcinoma. ${ }^{34,36,37}$ It has been postulated that MUC2 plays a major role in the indolent behavior of colloid carcinomas mainly by 'containing' the tumor cells and acting as a physical barrier against their spread. $^{31,38}$ Therefore, one can speculate that the reverse polarization in invasive micropapillary carcinoma coupled with the absence of the gelforming mucin MUC2 facilitates the secretion towards the stroma by the tumor cells of products responsible for stromal and vascular invasion namely metalloproteinases permitting 'easier' dissemination of the neoplastic cell clusters. This may explain the fact that the morphology of the tumor is retained in lymphovascular spaces as well as in lymph nodes and distant metastatic sites. It could also explain why tumors with this particular morphology, even when small in size, display a higher propensity for lymph node metastases. ${ }^{7}$ Such hypothesis, however, would not explain the behavior of invasive micropapillary carcinoma arising in the ovary, in the pathogenesis of which other mechanisms might be implicated.

It would be interesting to study the behavior and the expression of MUC1 and MUC2 glycoproteins in those invasive micropapillary carcinomas that also have a mucinous component (ie mixed colloid pattern). One earlier study briefly addressed this issue showing that tumors of patients with breast invasive micropapillary carcinoma that were disease free after a mean follow-up of approximately 5 years commonly had a colloid pattern. ${ }^{3}$

In summary, invasive micropapillary carcinoma is characterized by a reversal of cellular polarization and expression of MUC1 in the stroma-facing surface of the cells which may be one of the key factors in the distinctive morphology of this tumor type by causing the detachment of the neoplastic cells from the stroma, enhancing their spread and leading to early dissemination to lymph nodes. 


\section{References}

1 Siriaunkgul S, Tavassoli FA. Invasive micropapillary carcinoma of the breast. Mod Pathol 1993;6:660-662.

2 Luna-More S, Gonzalez B, Acedo C, et al. Invasive micropapillary carcinoma of the breast. A new special type of invasive mammary carcinoma. Pathol Res Pract 1994;190:668-674.

3 Luna-More S, de los Santos F, Breton JJ, et al. Estrogen and progesterone receptors, c-erbB-2, p53, and Bcl-2 in thirty-three invasive micropapillary breast carcinomas. Pathol Res Pract 1996;192:27-32.

4 Middleton LP, Tressera F, Sobel ME, et al. Infiltrating micropapillary carcinoma of the breast. Mod Pathol 1999;12:499-504.

5 Tresserra F, Grases PJ, Fabregas R, et al. Invasive micropapillary carcinoma. Distinct features of a poorly recognized variant of breast carcinoma. Eur J Gynaecol Oncol 1999;20:205-208.

6 Nassar H, Wallis T, Andea A, et al. Clinicopathologic analysis of invasive micropapillary differentiation in breast carcinoma. Mod Pathol 2001;14:836-841.

7 Walsh MM, Bleiweiss IJ. Invasive micropapillary carcinoma of the breast: eighty cases of an underrecognized entity. Hum Pathol 2001;32:583-589.

8 Ligtenberg MJ, Vos HL, Gennissen AM, et al. Episialin, a carcinoma-associated mucin, is generated by a polymorphic gene encoding splice variants with alternative amino termini. J Biol Chem 1990;265: 5573-5578.

9 Gendler SJ, Lancaster CA, Taylor-Papadimitriou J, et al. Molecular cloning and expression of human tumorassociated polymorphic epithelial mucin. J Biol Chem 1990;265:15286-15293.

10 Swallow DM, Gendler S, Griffiths B, et al. The hypervariable gene locus PUM, which codes for the tumour associated epithelial mucins, is located on chromosome 1, within the region 1q21-24. Ann Hum Genet 1987;51(Part 4):289-294.

11 Amin MB, Ro JY, el-Sharkawy T, et al. Micropapillary variant of transitional cell carcinoma of the urinary bladder. Histologic pattern resembling ovarian papillary serous carcinoma. Am J Surg Pathol 1994;18: 1224-1232.

12 Smith Sehdev AE, Sehdev PS, Kurman RJ. Noninvasive and invasive micropapillary (low-grade) serous carcinoma of the ovary: a clinicopathologic analysis of 135 cases. Am J Surg Pathol 2003;27:725-736.

13 Amin MB, Tamboli P, Merchant SH, et al. Micropapillary component in lung adenocarcinoma: a distinctive histologic feature with possible prognostic significance. Am J Surg Pathol 2002;26:358-364.

14 Winterford CM, Walsh MD, Leggett BA, et al. Ultrastructural localization of epithelial mucin core proteins in colorectal tissues. J Histochem Cytochem 1999;47:1063-1074.

15 Gendler SJ, Spicer AP, Lalani EN, et al. Structure and biology of a carcinoma-associated mucin, MUC1. Am Rev Respir Dis 1991;144:S42-S47.

16 Lan MS, Batra SK, Qi WN, et al. Cloning and sequencing of a human pancreatic tumor mucin cDNA. J Biol Chem 1990;265:15294-15299.

17 Hilkens J, Vos HL, Wesseling J, et al. Is episialin/MUC1 involved in breast cancer progression? Cancer Lett 1995;90:27-33.

18 Wesseling J, van der Valk SW, Vos HL, et al. Episialin (MUC1) overexpression inhibits integrin-mediated cell adhesion to extracellular matrix components. J Cell Biol 1995;129:255-265.

19 Wesseling J, van der Valk SW, Hilkens J. A mechanism for inhibition of E-cadherin-mediated cell-cell adhesion by the membrane-associated mucin episialin/ MUC1. Mol Biol Cell 1996;7:565-577.

20 McDermott KM, Crocker PR, Harris A, et al. Overexpression of MUC1 reconfigures the binding properties of tumor cells. Int J Cancer 2001;94:783-791.

21 Hudson MJ, Stamp GW, Chaudhary KS, et al. Human MUC1 mucin: a potent glandular morphogen. J Pathol 2001;194:373-383.

22 Ellis IO, Hinton CP, MacNay J, et al. Immunocytochemical staining of breast carcinoma with the monoclonal antibody NCRC 11: a new prognostic indicator. Br Med J (Clin Res Ed) 1985;290:881-883.

23 Ellis IO, Bell J, Todd JM, et al. Evaluation of immunoreactivity with monoclonal antibody NCRC 11 in breast carcinoma. Br J Cancer 1987;56:295-299.

24 Muir IM, Reed RG, Stacker SA, et al. The prognostic value of immunoperoxidase staining with monoclonal antibodies NCRC-11 and 3E1.2 in breast cancer. Br J Cancer 1991;64:124-127.

25 Walker RA. Assessment of milk fat globule membrane antibodies and lectins as markers of short-term prognosis in breast cancer. Br J Cancer 1990;62:462-466.

26 Rahn JJ, Dabbagh L, Pasdar M, et al. The importance of MUC1 cellular localization in patients with breast carcinoma: an immunohistologic study of 71 patients and review of the literature. Cancer 2001;91: 1973-1982.

27 McGuckin MA, Walsh MD, Hohn BG, et al. Prognostic significance of MUC1 epithelial mucin expression in breast cancer. Hum Pathol 1995;26:432-439.

28 Hayes DF, Mesa-Tejada R, Papsidero LD, et al. Prediction of prognosis in primary breast cancer by detection of a high molecular weight mucin-like antigen using monoclonal antibodies DF3, F36/22, and CU18: a Cancer and Leukemia Group B study. J Clin Oncol 1991;9:1113-1123.

29 Ohuchi N, Harada Y, Masuko T, et al. Characterization of cell surface antigens expressed in the HMA-1 breast cancer cell line. Surg Today 1995;25:244-250.

30 Ceriani RL, Chan CM, Baratta FS, et al. Levels of expression of breast epithelial mucin detected by monoclonal antibody BrE-3 in breast-cancer prognosis. Int J Cancer 1992;51:343-354.

31 Adsay NV, Merati K, Nassar H, et al. Pathogenesis of colloid (pure mucinous) carcinoma of exocrine organs: coupling of gel-forming mucin (MUC2) production with altered cell polarity and abnormal cell-stroma interaction may be the key factor in the morphogenesis and indolent behavior of colloid carcinoma in the breast and pancreas. Am J Surg Pathol 2003;27: 571-578.

32 Komaki K, Sakamoto G, Sugano H, et al. Mucinous carcinoma of the breast in Japan. A prognostic analysis based on morphologic features. Cancer 1988;61: 989-996.

33 Clayton F. Pure mucinous carcinomas of breast: morphologic features and prognostic correlates. Hum Pathol 1986;17:34-38.

34 Adsay NV, Pierson C, Sarkar F, et al. Colloid (mucinous noncystic) carcinoma of the pancreas. Am J Surg Pathol 2001;25:26-42.

35 Seidel G, Zahurak M, Iacobuzio-Donahue C, et al. Almost all infiltrating colloid carcinomas of the 
1050

pancreas and periampullary region arise from in situ papillary neoplasms: a study of 39 cases. Am J Surg Pathol 2002;26:56-63.

36 Ho SB, Niehans GA, Lyftogt C, et al. Heterogeneity of mucin gene expression in normal and neoplastic tissues. Cancer Res 1993;53:641-651.

37 O’Connell JT, Shao ZM, Drori E, et al. Altered mucin expression is a field change that accompanies muci- nous (colloid) breast carcinoma histogenesis. Hum Pathol 1998;29:1517-1523.

38 Adsay NV, Merati K, Andea A, et al. The dichotomy in the preinvasive neoplasia to invasive carcinoma sequence in the pancreas: differential expression of MUC1 and MUC2 supports the existence of two separate pathways of carcinogenesis. Mod Pathol 2002;15:1087-1095. 